\title{
Clinical assessment of patients with Graves' orbitopathy: the European Group on Graves' Orbitopathy recommendations to generalists, specialists and clinical researchers
}

The European Group on Graves' Orbitopathy (EUGOGO): W M Wiersinga ${ }^{1}$, P Perros ${ }^{3}$, G J Kahaly ${ }^{4}$, M P Mourits ${ }^{2}$, L Baldeschi $^{2}$, K Boboridis ${ }^{5}$, A Boschi ${ }^{6}$, A J Dickinson ${ }^{7}$, P Kendall-Taylor ${ }^{8}$, G E Krassas ${ }^{9}$, C M Lane ${ }^{10}$, J H Lazarus ${ }^{11}$,

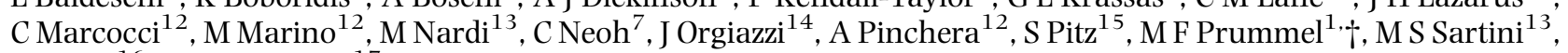
$\mathrm{M} \mathrm{Stahl}^{16}$ and G von Arx ${ }^{17}$

Departments of ${ }^{1}$ Endocrinology and ${ }^{2}$ Ophthalmology, Orbital Centre, Academic Medical Centre, Amsterdam, The Netherlands, ${ }^{3}$ Department of Endocrinology, Freeman Hospital, Newcastle upon Tyne, UK, ${ }^{4}$ Department of Medicine I, Gutenberg University Hospital, Mainz, Germany, ${ }^{5}$ University Department of Ophthalmology, Ahepa Hospital, Thessaloniki, Greece, ${ }^{6}$ Department of Ophthalmology, Universite Catholique de Louvain, Brussels, Belgium, ${ }^{7}$ Department of Ophthalmology, Royal Victoria Infirmary, Newcastle upon Tyne, UK, ${ }^{8}$ Medical School, University of Newcastle upon Tyne, Newcastle upon Tyne, UK, ${ }^{9}$ Department of Endocrinology, Panagia General Hospital, Thessaloniki, Greece, ${ }^{10}$ Cardiff Eye Unit, University Hospital of Wales, Heath Park, Cardiff, UK, ${ }^{11}$ School of Medicine, Cardiff University, Llandough Hospital, Cardiff, UK, Departments of ${ }^{12}$ Endocrinology and ${ }^{13}$ Neuroscience Section of Ophthalmology, University of Pisa, Pisa, Italy, ${ }^{14}$ Service of Endocrinology, Centre Hospitalier Lyon-Sud, Lyon, France, ${ }^{15}$ Department of Ophthalmology, Johannes Gutenberg-University, Mainz, Germany, ${ }^{16}$ Department of Internal Medicine and Endocrinology, Basedowch, Kantonsspital, Olten, Switzerland and ${ }^{17}$ Basedowch, ADMEDICO Augenzentrum, Olten, Switzerland

(Correspondence should be addressed to P Perros who is now at Endocrine Unit, Level 6, Freeman Hospital, Freeman Road, Newcastle upon Tyne NE7 7DN, UK; Email: petros.perros@ncl.ac.uk)

†Deceased.

\section{Introduction}

The European Group on Graves' Orbitopathy (EUGOGO) was established in order to promote better clinical care of patients with Graves' orbitopathy $(\mathrm{GO})(1,2)$ and to facilitate research in this disease. EUGOGO believes that patients with GO should be managed in multidisciplinary clinics with input from endocrinologists and ophthalmologists, who have an interest and experience in managing this disease (3). Although the expertise of the clinicians is critical and the setting perhaps less so, evidence from other clinical disciplines would suggest that a dedicated multidisciplinary clinic results in better outcomes $(4,5)$. EUGOGO is aware that many patients with GO in Europe never reach specialist centres or are referred too late to benefit from treatments (6), which results in a suboptimal outcome and sometimes loss of vision. EUGOGO recommends that primary care physicians, general practitioners, general internists and specialists, who have no particular expertise in managing such patients, should refer all but the mildest cases of GO to tertiary centres with established multidisciplinary clinics for further assessment and management. Selecting which patients should be referred and with what degree of urgency, is challenging for the generalist. EUGOGO proposes a simple clinical tool, which can be completed in less than 5 minutes by any medically qualified person and can help to identify patients who merit urgent or routine referral (Table 1 ).

\section{Evolution of recommendations}

Since 1999, EUGOGO has convened 11 meetings attended by endocrinologists and ophthalmologists from 12 major European centres all with extensive experience in managing patients with this condition. Several hundreds of 'man-hours' were dedicated to debating methods of assessment of patients with GO, including sessions using patients with various degrees of severity and activity, examined and scored by EUGOGO members. Based on this experience, EUGOGO has reached consensus on assessment of patients with GO and has embarked on randomised controlled clinical trials. EUGOGO believes that clinical examination is the cornerstone of the assessment of GO; clinically and scientifically meaningful data can be derived from careful, clearly defined clinical evaluations. Imaging of the orbits by CT or MRI scanning is recommended when the diagnosis is uncertain, in cases of apparently unilateral disease and suspected dysthyroid optic neuropathy (7). The role of serial orbital imaging by CT or MRI scanning in randomised controlled trials is uncertain, as the clinical importance of orbital volume changes is not yet defined and EUGOGO does not recommend these measurements as primary outcomes.

\section{Assessments in routine clinical practice}

The EUGOGO recommendations (Table 2) for assessing patients with GO are based on those of the sister thyroid societies published more than a decade ago (8) and 
apply both to routine clinical practice in the setting of specialist multidisciplinary clinics and to clinical research purposes. There are four principal advances compared to the previous recommendations of the sister thyroid societies: (a) in order to standardise the assessments of the subjective components of GO (soft tissue and inflammatory features), EUGOGO recommends specific methodology. This involves an examination protocol with a comparative photographic Colour Atlas $(9,10)$, which provides precise definitions for clinical signs and examples for grading their severity. Comparisons of the assessments and scores of several patients between EUGOGO members on the same day led to the conclusion that some features can be accurately categorised into more than one grade of severity, while others can only be reliably scored as 'present' or 'absent'. (b) A validated disease-specific questionnaire for assessment of quality of life is included $(10,11)$. (c) Although statistical comparisons of individual severity or activity parameters between or within patient groups may be scientifically interesting and worthwhile, EUGOGO recommends an overall evaluation for clinical trials, as this is more clinically

Table 1 Clinical tool for generalists to aid selection of patients with GO who should be referred to a specialist centre.

Patients with a history of Graves' disease, who have neither symptoms nor signs of $\mathrm{GO}$, require no further ophthalmological assessments and need not be referred to a specialist. Patients with unusual presentations (unilateral GO or euthyroid GO) should be referred however mild their symptoms or signs in order to make an accurate diagnosis. All other cases should be screened according to the protocol below:

Refer urgently if the answer is 'yes' to any of the following, particularly if onset is recent:

Symptoms

Unexplained deterioration in vision ${ }^{a}$

Awareness of change in intensity or quality of colour vision in one or both eyes? ${ }^{\mathrm{a}}$

History of eye(s) suddenly 'popping out'? (Globe subluxation)

Signs

Obvious corneal opacity?

Papilloedema? ${ }^{a}$

Refer non-urgently if the answer is 'yes' to any of the

following:

Symptoms

Eyes abnormally sensitive to light: troublesome or deteriorating over the past 1-2 months?

Eyes excessively gritty and not improving after 1 week of artificial eye drops?

Pain in or behind the eyes: troublesome or deteriorating over the past 1-2 months?

Appearance of the eyes and/or eyelids has changed over the past 1-2 months?

Appearance of the eyes is causing concern to the patient?

Can see two separate images when there should only be one and if so, is this troublesome or deteriorating?

Signs

Troublesome eyelid retraction?

Abnormal swelling or redness of eyelid(s) or conjunctiva(e)?

Restriction of eye movements?

Tilting of the head to avoid double vision?

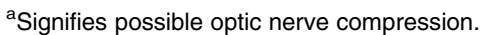

meaningful and relevant. Primary outcomes may usefully be divided into objective and subjective categories as the relationship between the two is complex but equally important (Table 3). (d) EUGOGO defines the overall beneficial response in an individual patient as an improvement of the objective parameter most relevant to the study in at least one eye without deterioration of any objective parameter in either eye. EUGOGO recognises that the overall outcome is influenced by several factors, including whether the intervention is medical or surgical as well as the selection criteria of the study population. Therefore, the above definitions should be viewed as a model that may be usefully adapted for any study design.

\section{The future}

The management of patients with GO can be improved by properly designed and executed large-scale studies with adequate statistical power. The feasibility of rapid recruitment for multicentre trials has already been demonstrated (12). EUGOGO believes that the available

Table 2 European Group on Graves' Orbitopathy (EUGOGO) recommendations for assessment of GO in routine clinical practice in specialist multidisciplinary clinics and for clinical trials.

(a) Activity measures based on the classical features of inflammation (10): clinical activity score (CAS) (13). The final score (maximum 7 ) is the sum of all items present

Spontaneous retrobulbar pain

Pain on attempted up- or down gaze

Redness of the eyelids

Redness of the conjunctiva

Swelling of the eyelids

Inflammation of the caruncle and/or plica

Conjunctival oedema

(b) Severity measures

Lid aperture (distance between the lid margins in $\mathrm{mm}$ with the patient looking in the primary position, sitting relaxed and with distant fixation)

Swelling of the eyelids (absent, mild, moderate, severe) (10)

Redness of the eyelids (absent, present) (10)

Redness of the conjunctivae (absent, mild, moderate, severe)

(10)

Conjunctival oedema (absent, present) (10)

Inflammation of the caruncle or plica (absent, present) (10)

Exophthalmos (measured in $\mathrm{mm}$ using the same Hertel exophthalmometer and same intercanthal distance for an individual patient)

Subjective diplopia score (0, no diplopia; 1 , intermittent, i.e. diplopia in primary position of gaze, when tired or when first awakening; 2, inconstant, i.e. diplopia at extremes of gaze; 3 , constant, i.e. continuous diplopia in primary or reading position) Eye muscle involvement (ductions in degrees) (10)

Corneal involvement (absent/punctate keratopathy/ulcer)

Optic nerve involvement

Best corrected visual acuity

Colour vision

Visual fields (to be included only if optic nerve compression is suspected)

Optic disc

Relative afferent pupillary defect (absent/present) 
Table 3 EUGOGO recommendations for assessing response to intervention in clinical trials.

\begin{tabular}{ll}
\hline Primary outcomes & \\
\hline Objective parameters & Change required \\
CAS & $\geq 2$ Points \\
Lid aperture & $\geq 2 \mathrm{~mm}$ \\
Soft tissue involvement & $\geq$ One grade in any of the following: \\
& Eyelid swelling, eyelid erythema, conjunctival redness or conjunctival oedema \\
Exophthalmos & $\geq 2 \mathrm{~mm}$ \\
Subjective diplopia & $\geq$ One grade \\
Ductions & $\geq 8^{\circ}$ In at least one direction of gaze \\
Visual function & Change of best corrected visual acuity by $\geq 2$ lines on Snellen chart, or \\
& Substantial colour vision change, or \\
& Significant change of visual fields, or \\
& Significant change in optic disc appearance, or \\
Subjective parameters & (Dis-)appearance of relative afferent pupillary defect \\
Disease-specific quality-of-life questionnaire & \\
(GO-QOL) (10, 11) & \\
Visual functioning (score 0-100) & $\geq 6$ Points \\
Appearance (score $0-100)$ & $\geq 6$ Points \\
\hline
\end{tabular}

\section{Secondary outcome}

Orbital volume changes by serial imaging may be included as a secondary outcome, although the clinical importance of volume changes remain to be defined

The left hand column lists the parameters assessed; the right hand column defines the minimum change required in each parameter for an individual patient before and after an intervention, for the purposes of classifying the overall response.

methodology as outlined here can be used for clinical trials as well as for the routine clinical assessment of patients with GO and therefore will be of interest to those who manage patients with this condition.

\section{The European Group on Graves' Orbitopathy}

EUGOGO is a multidisciplinary consortium of clinicians from European centres, who share a commitment to improve the management of GO, have a good track record in clinical research in this field, are regional or national referral centres for the treatment of this disease and manage patients in a multidisciplinary setting with input from endocrinologists and ophthalmologists.

\section{References}

1 Bartalena L, Pinchera A \& Marcocci C. Management of Graves' ophthalmopathy: reality and perspectives. Endocrine Reviews 2000 21 168-199.

2 Kahaly GJ, Petrak F, Hardt J, Pitz S \& Egle UT. Psychosocial morbidity of Graves' orbitopathy. Clinical Endocrinology 200563 395-402.

3 Wiersinga WM. The philosophy of Graves' ophthalmopathy. Orbit 200524 165-171.

4 Ducharme A, Doyon O, White M, Rouleau JL \& Brophy JM. Impact of care at a multidisciplinary congestive heart failure clinic: a randomized trial. Cancer Medical Associtation Journal 2005173 40-45.

5 Sperl-Hillen JM \& O'Connor PJ. Factors driving diabetes care improvement in a large medical group: 10 years of progress. American Journal of Management Care 20055 S177-S185.
6 Perros P, Baldeschi L, Boboridis K, Dickinson AJ, Hullo A, Kahaly GJ, Kendall-Taylor P, Krassas GE, Lane CM, Lazarus JH, Marcocci C, Marino M, Mourits MP, Nardi M, Orgiazzi J, Pinchera A, Pi S, Prummel MF, Wiersinga WM. A questionnaire survey on the management of Graves' orbitopathy in Europe. European Journal of Endocrinology 2006155 207-211.

7 Kahaly GJ. Imaging in thyroid-associated orbitopathy. European Journal of Endocrinology 2001145 107-118.

8 Pinchera A, Wiersinga WM, Glinoer D, Kendall-Taylor P, Koornneef L, Marcocci C, Schleusener H, Romaldini J, Niepominiscze $H$, Nagataki S, Izumi $M$, Inoue $Y$, Stockigt J, Wall J, Greenspan F, Soloman D, Garrity J \& Gorman A. Classification of eye changes of Graves' disease. Thyroid 19922 235-236.

9 Dickinson AJ \& Perros P. Controversies in the clinical evaluation of active thyroid-associated orbitopathy: use of a detailed protocol with comparative photographs for objective assessment. Clinical Endocrinology 200155 283-303.

10 www.eugogo.org

11 Terwee CB, Gerding MN, Dekker FW, Prummel MF \& Wiersinga WM. Development of a disease specific quality of life questionnaire for patients with Graves' ophthalmopathy: the GO-QOL. British Journal of Ophthalmology $1998 \mathbf{8 2} 773-779$.

12 Prummel MF, Bakker A, Wiersinga WM, Baldeschi L, Mourits MP, Kendall-Taylor P, Perros P, Neoh C, Dickinson AJ, Lazarus JH, Lane CM, Heufelder AE, Kahaly GJ, Pitz S, Orgiazzi J, Hullo A, Pinchera A, Marcocci C, Sartini MS, Rocchi R, Nardi M, Krassas GE \& Halkias A. Multi-center study on the characteristics and treatment strategies of patients with Graves' orbitopathy: the first European Group on Graves' Orbitopathy experience. European Journal of Endocrinology 2003148 491-495.

13 Mourits MP, Prummel M, Wiersinga WM \& Koornneef L. Clinical activity score as a guide in the management of patients with Graves' ophthalmopathy. Clinical Endocrinology 1997 47 9-14.

Received 12 May 2006

Accepted 13 June 2006 